\title{
A High-Speed Low-Cost Millimeter Wave System with Dual Pulse Shaping Transmission and Symbol Rate Equalization Techniques
}

\author{
Hao Zhang, Xiaojing Huang, \\ Jian A. Zhang, Y. Jay Guo \\ Global Big Data Technologies Centre \\ University of Technology Sydney, Australia \\ Emails: \{Hao.Zhang, Xiaojing.Huang, \\ Andrew.Zhang, Jay.Guo\}@uts.edu.au
}

\author{
RuiLiang Song, \\ ChunTing Wang, Wei Wu \\ The 54th Research Institute of China \\ Electronics Technology \\ Group Corporation, China \\ Emails: songruiliang@hotmail.com,
} chunting.w@263.net, cetc_wuwei@163.com

\author{
XiaoFan Xu, \\ Zhou Lu \\ China Academy of Electronics and \\ Information Technology, China \\ Emails: xiaofanxu@live.com, \\ cetc_luzhou@163.com
}

\begin{abstract}
A millimeter wave system with commercially available and affordable data conversion devices is presented in this paper for achieving high-speed and low-cost wireless communications. By adopting the proposed dual pulse shaping (DPS) transmission scheme, the system can achieve full Nyquist rate transmission with only half of the sampling rate required by conventional Nyquist pulse shaping. Structures of the DPS transmitter and receiver are described and effective symbol rate equalization techniques suitable for DPS transmission are presented. Simulation results with two sets of practical dual spectral shaping pulses are also provided to compare system performance with the conventional Nyquist pulse shaping system.

Index Terms-High-speed, low-cost, millimeter wave, equalization and data conversion devices.
\end{abstract}

\section{INTRODUCTION}

With the ever increased demands for high data rate wireless communications, it is necessary to increase the signal bandwidth and improve the spectral efficiency at the same time [1]. For high-speed wideband wireless applications and aerial backbone networks, millimetre wave (mm-wave) communication system has been proven to be a viable solution since there is more bandwidth to be used for wireless transmission in mm-wave bands such as the E-band (71-76 and 81-86 $\mathrm{GHz}$ ) which has two $5 \mathrm{GHz}$ contiguous bands [2] [3]. In $110-170 \mathrm{GHz} \mathrm{D}$ band, more than $10 \mathrm{GHz}$ bandwidth is also available. On the other hand, the devices for digital-to-analog conversion (D/A) and analog-to-digital conversion (A/D) at high sampling rate such as 5 Giga samples per second (Gsps) or higher are lacking or very expensive. In order to achieve high-speed mm-wave communications with $5 \mathrm{GHz}$ or wider signal bandwidth, higher than 5 Gsps sampling rate D/A and $\mathrm{A} / \mathrm{D}$ devices must be used. When the required high-speed data conversion devices are unattainable or costly, how to achieve high date rate wireless communications at low cost becomes a significant technological challenge.

As we all know, inter-symbol interference (ISI) caused by frequency selective and multi-path channels will bring the unpredictable error at the receiver and degrade the system performance. It is always necessary to adopt suitable equalization techniques in the system design and implementation [4] [5]. To combat ISI, equalizers are commonly devised to either cancel it by zero-forcing (ZF) equalization or reduce it while avoiding noise enhancement by Minimum Mean Square Error (MMSE) equalization [6]. However, since conventional fractionally spaced equalization techniques require that the sampling rate must be higher than the data symbol rate, the requirement for high-speed data conversion devices is even more stringent. Higher sampling rate also leads to higher computational complexity for digital signal processing implementation.

In this paper, a high-speed mm-wave system implemented with only half symbol rate data conversion devices is proposed. It is achieved by employing a novel dual pulse shaping (DPS) transmission technique and adopting symbol rate equalization. With DPS transmission, the data symbols to be transmitted are split into two half rate data streams with each data stream being sampled by half of the symbol rate. Symbol rate equalizations are performed to the two data streams respectively at receiver to remove both the ISI in each stream and the cross-symbol interference (CSI) between them. Therefore, lower computation complexity and implementation cost can be achieved with reduced volume and power consumption for digital signal processing compared with conventional fractionally spaced equalization. The proposed system solves the problem currently facing the high-speed wideband communications due to the lack of affordable D/A and A/D at high sampling rate such as 5 Gsps or higher. Two sets of practical pulses suitable for DPS transmission are also proposed to verify the feasibility of the high-speed low-cost system implementation. System performance comparison in terms of peak-to-average power ratio (PAPR) and bit error rate (BER) with conventional single pulse shaping (SPS) is also performed through simulation.

The rest of this paper is organized as follows. In Section II, the DPS transmission system is described. In Section III, the symbol rate ZF and MMSE equalization techniques for the 
DPS transmission are formulated. Simulation results are given in Section IV to compare the performance between DPS and SPS. Finally, conclusions are drawn in Section V.

\section{System Description}

The block diagram of the DPS transmission system with half symbol rate D/As and A/Ds is shown in Fig. 1. The radio frequency (RF) chains (such as the up and down converters, high power amplifier and low noise amplifier, etc.) and antennas are not shown for simplicity.

At the transmitter side, the input data bits are firstly encoded and mapped into data symbols with symbol duration $T_{s}$. A serial-to-parallel converter $(\mathrm{S} / \mathrm{P})$ is then used to split the serial data symbol stream into two half-rate parallel data symbol streams. The two parallel data symbol streams are converted into analog signals by the D/As with sampling rate equal to half symbol rate. After passing through the two pulse shaping filters respectively, the two parallel data symbol streams form two separate spectrally shaped signals which are then combined and filtered by a transmitter (Tx) filter to form the transmitted baseband signal.

At the receiver side, the received baseband signal is firstly filtered by a receiver ( $\mathrm{Rx})$ filter. Then, two digital signal streams are obtained by sampling the received baseband signal using the A/Ds with sampling rate equal to half of the symbol rate, followed by up-sampling by a ratio of two. Note that the clock of the A/Ds for sampling the second data stream have $T_{s}$ delay. With the second data stream delayed by one sample, the combined digital signal stream is equalized by two equalization filters which operate at symbol rate and down sampled by a ratio of two respectively to recover the two streams of the transmitted data symbols. A parallel-toserial converter (P/S) merges the two data symbol streams into one serial data symbol stream, followed by de-mapping and decoding to retrieve the transmitted information bits.

\section{Equalization TechNologies}

\section{A. Received and Equalized Signal Models}

Suppose that the data symbols to be transmitted are represented by a discrete sequence $s(n)$. After $\mathrm{S} / \mathrm{P}$, the two parallel data symbol streams can be represented as $s_{1}\left(\frac{n}{2}\right)=\left\{\begin{array}{ll}s(n) & \text { for even } n \\ 0 & \text { for odd } n\end{array}\right.$ and $s_{2}\left(\frac{n}{2}\right)=\left\{\begin{array}{ll}s(n+1) & \text { for even } n \\ 0 & \text { for odd } n\end{array}\right.$ respectively. The Fourier transforms of the two parallel sequences can be expressed as $S_{1}\left(e^{j 2 \omega}\right)$ and $S_{2}\left(e^{j 2 \omega}\right)$ which are periodic functions of $\omega$ with period $\pi$. Furthermore, suppose that the frequency responses (the combinations of the respective pulse shaping filter, Tx filter, and $\mathrm{Rx}$ filter) for the DPS channel are $H_{1}(f)$ and $H_{2}(f)$ respectively and the noise is represented in frequency domain as $W(f)$. The received signal after the Rx filter in frequency domain is then expressed as

$$
R(f)=H_{1}(f) S_{1}\left(e^{j 4 \pi f T_{s}}\right)+H_{2}(f) S_{2}\left(e^{j 4 \pi f T_{s}}\right)+W(f),
$$

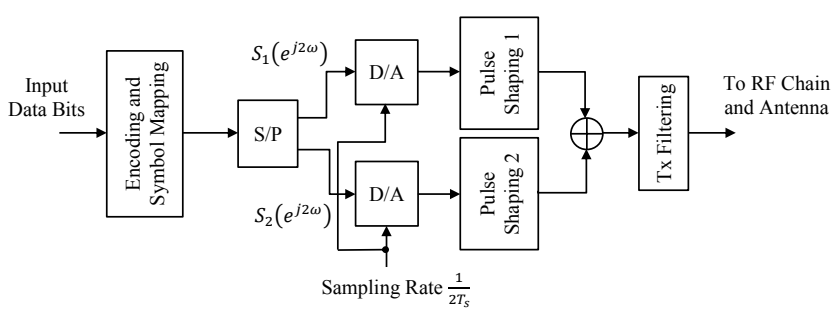

(a)

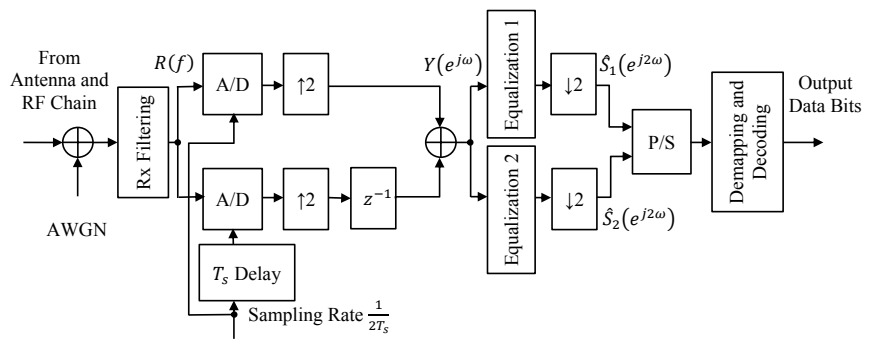

(b)

Fig. 1: Dual pulse shaping transmitter (a) and receiver (b).

where the relationship $\omega=2 \pi f T_{s}$ between digital frequency $\omega$ and analog frequency $f$ is used. Note that at the input of the A/Ds with $T_{s}$ clock delay, the signal will appear as a $T_{s}$ advanced version of $R(f)$ with frequency domain expression $e^{j 2 \pi f T_{s}} R(f)$.

The digital frequency domain received signal model can be expressed in matrix form as

$$
\mathbb{Y}(\omega)=\mathbb{H}(\omega) \mathbb{S}(\omega)+\mathbb{W}(\omega), \text { for } 0 \leq \omega<\pi,
$$

where $\mathbb{Y}(\omega)=\left[\begin{array}{c}Y\left(e^{j \omega}\right) \\ Y\left(e^{j(\omega-\pi)}\right)\end{array}\right], \mathbb{S}(\omega)=\left[\begin{array}{l}S_{1}\left(e^{j 2 \omega}\right) \\ S_{2}\left(e^{j 2 \omega}\right)\end{array}\right], \mathbb{H}(\omega)=$ $\left[\begin{array}{cc}\widetilde{H}_{1}\left(e^{j \omega}\right) & \widetilde{H}_{2}\left(e^{j \omega}\right) \\ \widetilde{H}_{1}\left(e^{j(\omega-\pi)}\right) & \widetilde{H}_{2}\left(e^{j(\omega-\pi)}\right)\end{array}\right]$ and $\mathbb{W}(\omega)=\left[\begin{array}{c}\widetilde{W}\left(e^{j \omega}\right) \\ \widetilde{W}\left(e^{j(\omega-\pi)}\right)\end{array}\right]$. $Y\left(e^{j \omega}\right)$ is the received signal frequency domain representation, $\widetilde{H}_{1}\left(e^{j \omega}\right)=\frac{1}{2 T_{s}} \sum_{k=-\infty}^{\infty} H_{1}\left(\frac{\omega}{2 \pi T_{s}}-k \frac{1}{T_{s}}\right)$, $\widetilde{H}_{2}\left(e^{j \omega}\right)=\frac{1}{2 T_{s}} \sum_{k=-\infty}^{\infty} H_{2}\left(\frac{\omega}{2 \pi T_{s}}-k \frac{1}{T_{s}}\right)$, and $\widetilde{W}\left(e^{j \omega}\right)=$ $\frac{1}{2 T_{s}} \sum_{k=-\infty}^{\infty} W\left(\frac{\omega}{2 \pi T_{s}}-k \frac{1}{T_{s}}\right)$.

Denoting the frequency responses of the two digital equalizers as $C_{1}\left(e^{j \omega}\right)$ and $C_{2}\left(e^{j \omega}\right)$ respectively, the equalized and down-sampled (by a factor of 2, leading to spectral overlapping) signals, which are the estimates of the transmitted signals, can be expressed in the frequency domain as

$$
\hat{S}_{1}\left(e^{j 2 \omega}\right)=C_{1}\left(e^{j \omega}\right) Y\left(e^{j \omega}\right)+C_{1}\left(e^{j(\omega-\pi)}\right) Y\left(e^{j(\omega-\pi)}\right)
$$

and

$$
\hat{S}_{2}\left(e^{j 2 \omega}\right)=C_{2}\left(e^{j \omega}\right) Y\left(e^{j \omega}\right)+C_{2}\left(e^{j(\omega-\pi)}\right) Y\left(e^{j(\omega-\pi)}\right),
$$

or, in matrix form

$$
\hat{\mathbb{S}}(\omega)=\left[\begin{array}{l}
\hat{S}_{1}\left(e^{j 2 \omega}\right) \\
\hat{S}_{2}\left(e^{j 2 \omega}\right)
\end{array}\right]=\left[\begin{array}{ll}
C_{1}\left(e^{j \omega}\right) & C_{1}\left(e^{j(\omega-\pi)}\right) \\
C_{2}\left(e^{j \omega}\right) & C_{2}\left(e^{j(\omega-\pi)}\right)
\end{array}\right] \mathbb{Y}(\omega)
$$

for $0 \leq \omega<\pi . C_{1}\left(e^{j \omega}\right)$ and $C_{2}\left(e^{j \omega}\right)$ can be determined according to the equalization techniques as discussed below. 
After converting $C_{1}\left(e^{j \omega}\right)$ and $C_{2}\left(e^{j \omega}\right)$ into time domain impulse responses respectively, the symbol rate equalizers can be implemented as time domain linear filters.

\section{B. ZF Equalization}

With ZF equalization, the transmitted signal can be recovered from (2) as

$\hat{\mathbb{S}}(\omega)=\left[\begin{array}{l}\hat{S}_{1}\left(e^{j 2 \omega}\right) \\ \hat{S}_{2}\left(e^{j 2 \omega}\right)\end{array}\right]=\mathbb{H}^{-1}(\omega) \mathbb{Y}(\omega)=\mathbb{S}(\omega)+\mathbb{H}^{-1}(\omega) \mathbb{W}(\omega)$.

The inverse channel matrix can be calculated as

$$
\mathbb{H}^{-1}(\omega)=\frac{\left[\begin{array}{cc}
\widetilde{H}_{2}\left(e^{j(\omega-\pi)}\right) & -\widetilde{H}_{2}\left(e^{j \omega}\right) \\
-\widetilde{H}_{1}\left(e^{j(\omega-\pi)}\right) & \widetilde{H}_{1}\left(e^{j \omega}\right)
\end{array}\right]}{\widetilde{H}_{1}\left(e^{j \omega}\right) \widetilde{H}_{2}\left(e^{j(\omega-\pi)}\right)-\widetilde{H}_{2}\left(e^{j \omega}\right) \widetilde{H}_{1}\left(e^{j(\omega-\pi)}\right)}
$$

and the equalizer frequency responses are obtained from the inverse channel matrix and using the relationship expressed in (5) as

$$
\begin{aligned}
& C_{1}\left(e^{j \omega}\right)= \\
& \left\{\begin{array}{l}
\frac{\widetilde{H}_{2}\left(e^{j(\omega-\pi)}\right)}{\widetilde{H}_{1}\left(e^{j \omega}\right) \widetilde{H}_{2}\left(e^{j(\omega-\pi)}\right)-\widetilde{H}_{2}\left(e^{j \omega}\right) \widetilde{H}_{1}\left(e^{j(\omega-\pi)}\right)}, 0 \leq \omega<\pi \\
\frac{-\widetilde{H}_{2}\left(e^{j(\omega+\pi)}\right)}{\widetilde{H}_{1}\left(e^{j(\omega+\pi)}\right) \widetilde{H}_{2}\left(e^{j \omega}\right)-\widetilde{H}_{2}\left(e^{j(\omega+\pi)}\right) \widetilde{H}_{1}\left(e^{j \omega}\right)},-\pi \leq \omega<0
\end{array}\right.
\end{aligned}
$$

and

$$
\begin{aligned}
& C_{2}\left(e^{j \omega}\right)= \\
& \begin{cases}\frac{-\widetilde{H}_{1}\left(e^{j(\omega-\pi)}\right)}{\widetilde{H}_{1}\left(e^{j \omega}\right) \widetilde{H}_{2}\left(e^{j(\omega-\pi)}\right)-\widetilde{H}_{2}\left(e^{j \omega}\right) \widetilde{H}_{1}\left(e^{j(\omega-\pi)}\right)}, & 0 \leq \omega<\pi \\
\frac{\widetilde{H}_{1}\left(e^{j(\omega+\pi)}\right)}{\widetilde{H}_{1}\left(e^{j(\omega+\pi)}\right) \widetilde{H}_{2}\left(e^{j \omega}\right)-\widetilde{H}_{2}\left(e^{j(\omega+\pi)}\right) \widetilde{H}_{1}\left(e^{j \omega}\right)}, & -\pi \leq \omega<0\end{cases}
\end{aligned}
$$

respectively.

After ZF equalization, the output SNR can be derived as $\gamma_{Z F}=\gamma \frac{4 \pi^{2}}{\int_{0}^{\pi}\|\mathbb{H}(\omega)\|^{2} d \omega \int_{0}^{\pi}\left\|\mathbb{H}^{-1}(\omega)\right\|^{2} d \omega}$ where $\gamma$ is the SNR at the receiver input.

\section{MMSE Equalization}

With MMSE equalization, the equalizer frequency responses are obtained from the inverse channel matrix $\mathbb{G}(\omega)$ for $0 \leq \omega$ $<\pi$ defined as

$$
\mathbb{G}(\omega)=\left[\begin{array}{ll}
G_{11}(\omega) & G_{12}(\omega) \\
G_{21}(\omega) & G_{22}(\omega)
\end{array}\right]=\mathbb{H}^{H}(\omega)\left(\mathbb{H}(\omega) \mathbb{H}^{H}(\omega)+\frac{1}{\rho} \mathbb{I}\right)^{-1}
$$

where $(.)^{H}$ stands for matrix conjugation and transposition, $\rho$ is the ratio of signal power at transmitter to noise power at receiver, and $\mathbb{I}$ is the identity matrix of order two. The equalizer frequency responses are

$$
C_{1}\left(e^{j \omega}\right)= \begin{cases}G_{11}(\omega), & 0 \leq \omega<\pi \\ G_{12}(\omega+\pi), & -\pi \leq \omega<0\end{cases}
$$

and

$$
C_{2}\left(e^{j \omega}\right)= \begin{cases}G_{21}(\omega), & 0 \leq \omega<\pi \\ G_{22}(\omega+\pi), & -\pi \leq \omega<0 .\end{cases}
$$

Since the SNR at the receiver input can be evaluated as $\gamma=\rho \frac{\int_{0}^{\pi}\|\mathbb{H}(\omega)\|^{2} d \omega}{2 \pi}$, the output SNR after MMSE equalization can be derived as $\gamma_{M M S E}=\frac{2 \pi}{\int_{0}^{\pi} \operatorname{tr}\left\{\mathbb{I}-\mathbb{H}^{H}(\omega)\left(\mathbb{H}(\omega) \mathbb{H}^{H}(\omega)+\frac{\int_{0}^{\pi}\|\mathbb{H}(\omega)\|^{2} d \omega}{2 \pi \gamma} \mathbb{I}\right)^{-1} \mathbb{H}(\omega)\right\} d \omega}$ where $\operatorname{tr}\{$.$\} stands for the trace of a matrix.$

\section{Simulation Results}

To demonstrate the performance of the proposed DPS transmission system for high-speed low-cost mm-wave communications, we now present the simulation results assuming a 25 Gbps system with $5 \mathrm{GHz}$ bandwidth operating in the 71-76/81$86 \mathrm{GHz}$ E-band. The sampling rate required for this system is only 2.5 Gsps which is easily achieved with commercial A/D and D/A devices at low cost. The 64-ary quadrature amplitude modulation (64-QAM) is used to provide $6 \mathrm{bps} / \mathrm{Hz}$ spectral efficiency over $5 \mathrm{GHz}$ bandwidth, achieving $30 \mathrm{Gbps}$ raw data rate including some necessary overhead for synchronization, channel estimation, and other system functionalities.

Two schemes of practical spectral shaping pulse selection are proposed. The first scheme uses a return-to-zero rectangular pulse and its $T_{s}$ delayed version for DPS. After Tx filtering with a 10th order Butterworth lowpass filter, the two spectral shaping pulses are shown in Fig. 2. The second scheme uses a non-return-to-zero pulse and a Manchester pulse. The spectral shaping pulses after the same Tx filter are shown in Fig. 3. The frequency responses of the two pulses for each scheme are shown in Fig. 4. For comparison purpose, a root raisedcosine (RRC) spectrum with roll-off factor $\beta=0.15$ used for conventional Nyquist pulse shaping is also shown.

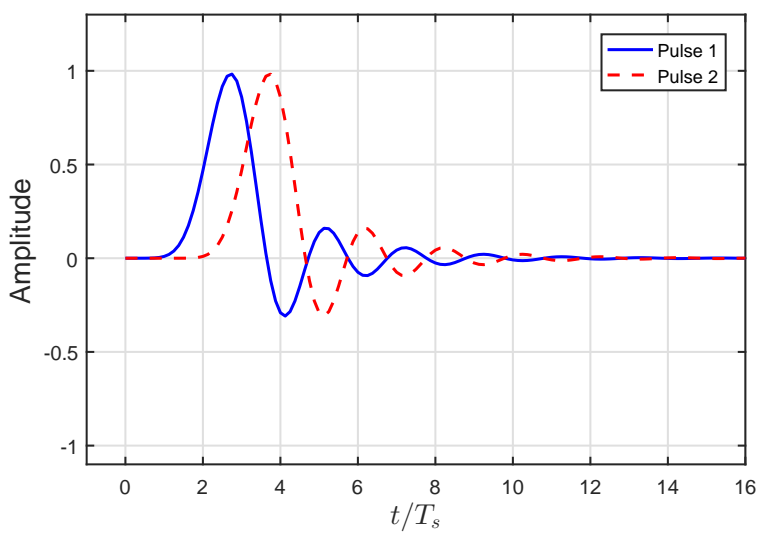

Fig. 2: Dual pulse shaping scheme 1.

We first compare the PAPR performance between DPS and SPS with Nyquist pulse, and the results are shown in Fig. 5. We see that the SPS generally has better PAPR performance than DPS. However, the degradation is insignificant for the proposed DPS scheme 1 .

We then compare the BER performance (uncoded) between the two pulse shaping schemes using ZF and MMSE equalizations respectively. As seen from Fig. 6, both pulse shaping schemes demonstrate almost the same performance in 


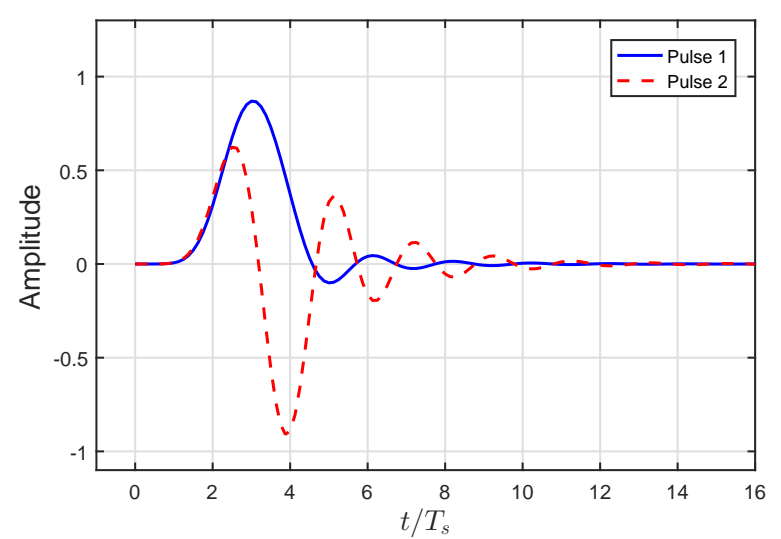

Fig. 3: Dual pulse shaping scheme 2 .

Gaussian channel with only additive white Gaussian noise and slight degradation as compared with that of SPS for both ZF and MMSE equalizations. In multipath fading channel where a two-ray model is assumed and the second path has random reflection with $3 \mathrm{~dB}$ lower average power and a delay of $6 \mathrm{~ns}$, the DPS scheme 2 demonstrates better performance than that of scheme 1 and approaches the SPS performance.

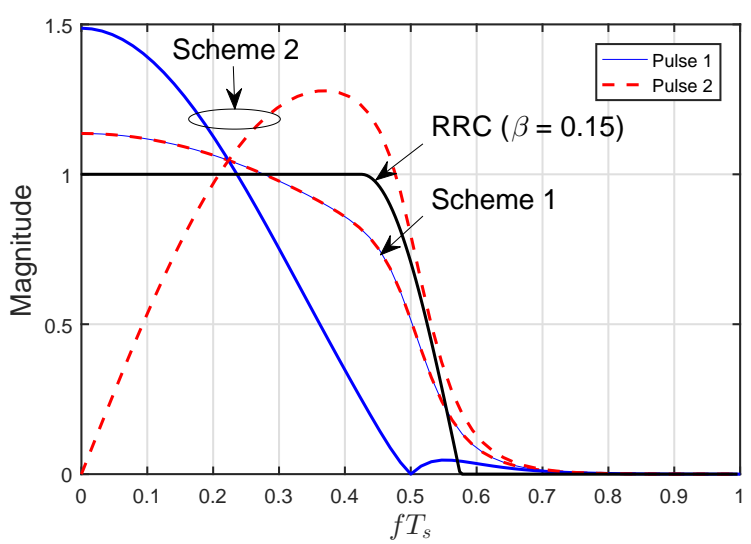

Fig. 4: Dual pulse shaping and RRC spectra.

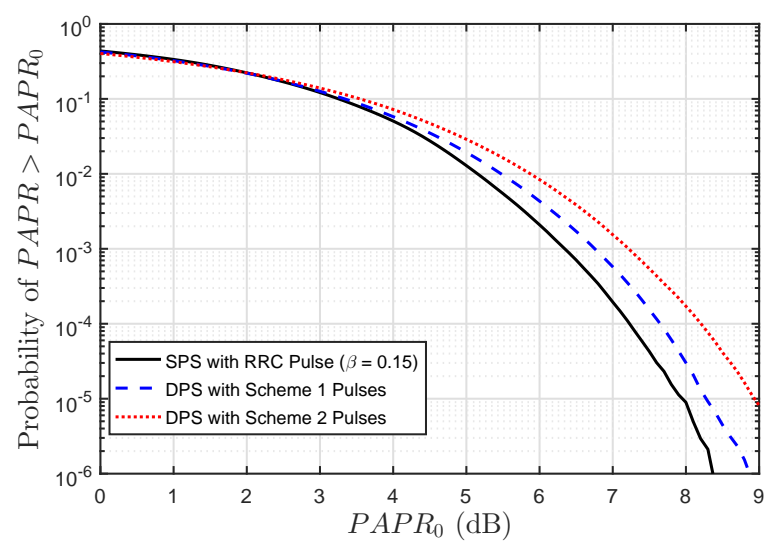

Fig. 5: PAPR comparison.

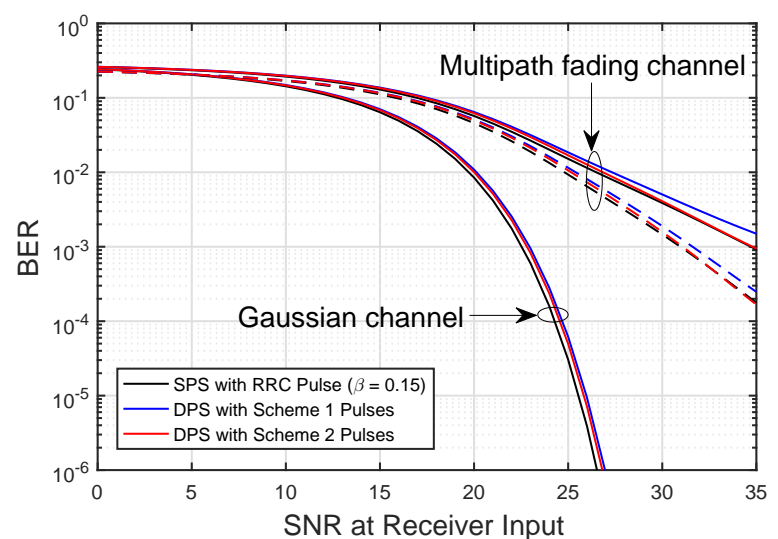

Fig. 6: BER comparison (solid lines for ZF equalization and dashed lines for MMSE equalization).

\section{CONCLUSION}

We have shown that the DPS transmission transmitter and receiver can be realized using D/As and A/Ds with sampling rate equal to only half of data symbol rate. The ZF and MMSE equalization techniques allow for practical dual spectral shaping pulses to be used. Two sets of practical pulses are proposed and simulated to verify the feasibility of the DPS transmission and equalization techniques. Simulation results show that the DPS transmission system can provide the equivalent BER performance with slight degradation on PAPR. When required data conversion devices are unattainable or costly, high-speed and low-cost wireless communication systems can be still achieved using the proposed DPS transmission and symbol rate equalization techniques with negligible performance loss.

\section{REFERENCES}

[1] J. G. Andrews, S. Buzzi, W. Choi, S. V. Hanly, A. Lozano, A. C. K. Soong, and J. C. Zhang, "What will 5G be?" IEEE Journal on Selected Areas in Communications, vol. 32, no. 6, pp. 1065-1082, June 2014.

[2] T. K. Woodward, "What to do when there is no fiber: the DARPA $100 \mathrm{~Gb} / \mathrm{s}$ RF backbone program," in 2017 Optical Fiber Communication Conference, CA, USA, 2017.

[3] X. Huang, "The mobile aerial backbone links for air-space-ground integrated information networks," in Journal of China Computer Federation, vol. 12, no. 3, March 2016, pp. 27-32.

[4] A. Coskun and I. Kale, "All-adaptive blind matched filtering for the equalization and identification of multipath channels - A practical approach," IEEE Transactions on Circuits and Systems I: Regular Papers, vol. 60, no. 1, pp. 232-242, Jan 2013.

[5] H. Huang, M. Pan, and S. Gong, "Estimating and calibrating the response of multiple wideband digital radio frequency memories in a hardware-inthe-loop system using shuffled frog leaping algorithm," IET Radar, Sonar Navigation, vol. 10, no. 5, pp. 827-833, 2016.

[6] H. Gazzah, "A note on optimum MMSE/ZF multichannel equalization delay," in 2010 17th International Conference on Telecommunications, April 2010, pp. 919-922. 